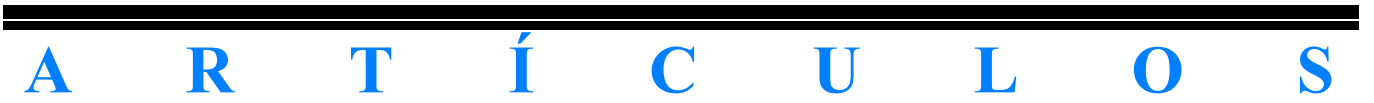




\section{SOBRE ALGUNAS LÓGICAS \\ PARACLÁSICAS Y EL ANÁLISIS \\ DEL RAZONAMIENTO JURÍDICO}

\section{Introducción}

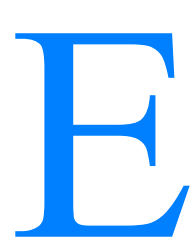

n esta nota exponemos una forma nueva de encarar sistemas proposicionales con contradicciones o inconsistencias. La misma, cuando se trata de sistemas consistentes, coincide con el tratamiento clásico. En realidad, lo que haremos es cambiar el concepto de deducción de la lógica clásica con el fin de poder manejar mejor teorías o sistemas proposicionales que incluyan contradicciones. De esta suerte somos llevados de hecho a construir una nueva categoría de lógica paraconsistente (cf. Arruda 1980). Esta lógica, que involucra a la lógica clásica como un caso particular, lógica clásica con la que está muy emparentada, será denominada, adoptando la terminología sugerida por V. S. Subrahmanian, «lógica paraclásica». Esta, en realidad, constituye un nuevo tipo de lógica paraconsistente.

La lógica paraclásica pareciera contar con numerosas aplicaciones, como, por ejemplo, en la informática y en el discurso normativo. Este último puede ser estudiado descriptiva o prescriptivamente, aunque aquí nos limitaremos a ese discurso en sus aspectos descriptivos. Reconocemos que esta limitación suscita algunos problemas, en tanto valga la tesis de que sólo el discurso prescriptivo consistente admite propiedades lógicas isomórficas con el discurso descriptivo correlativo, tal como lo han propuesto von Wright y Alchourrón (von Wright 1983 y Alchourrón 1969). Esta tesis ha sido utilizada para rescatar, entre otras cosas, la propuesta de $\mathrm{H}$. Keisen de que las relaciones lógicas que se dan en el discurso normativo descriptivo valen sólo analógicamente con las relaciones lógicas que atribuimos al discurso puramente prescriptivo. El desarrollo posterior de lógicas directamente construidas sobre el discurso normativo prescriptivo permiten hacerse cargo, como los juristas y moralistas efectivamente lo hacen, de la existencia de normas o prescripciones inconsistentes, como las que exhiben habitualmente

\footnotetext{
* Con colaboración especial de Leila Z. Puga (Sao Paulo)
} 
códigos normativos jurídicos positivos e inclusive las llamadas moralidades positivas. Ello, con prescindencia de las inconsistencias que puedan encontrarse entre las descripciones de esas normas que ensayarían, según se dice, ciencias como la jurisprudencia dogmática. Correspondería distinguir así entre el discurso normativo prescriptivo y el discurso metanormativo correspondiente. Este último carece en principio de funciones prescriptivas, aunque muchas veces los juristas y los moralistas, al sentar criterios de consistencia y sistematicidad para los códigos normativos que proponen o que defienden, recurren a un lenguaje metanormativo prescriptivo. Vale decir que, en estos casos, la aceptación de una lógica conlleva la adopción tácita de prescripciones metateóricas.

En lo que hace a la informática, todo indica que una lógica paraclásica encontraría aplicación en sistemas expertos y en programación lógica, cuando haya que recurrir a bases de datos o a informaciones inconsistentes.

En especial, en el campo jurídico, la toma de decisiones con asistencia de sistemas expertos, debe enfrentar informaciones sobre hechos y sobre normas prima facie contradictorias, como es el caso en cualquier situación litigiosa. De ahí los inconvenientes que resultarían de aplicar procedimientos deductivos clásicos a datos inconsistentes.

Nos ocuparemos en lo que sigue, sin embargo, del discurso normativo en general, cuyo problema fundamental pareciera ser el siguiente: dado un cierto código normativo (un conjunto de prescripciones o normas positivas, por ejemplo), cómo obtener alguna lógica que permita justificar deducciones e inferencias a partir de él, sin incurrir en trivialidad, aún cuando el código sea inconsistente. Naturalmente, el código mismo no será modificado, para hacerlo consistente, eliminando las contradicciones, operación que transformaría su naturaleza misma, aunque en ello consiste buena parte de la labor argumentativa de los jueces. Este tipo de transformación o cambio de un código ha sido propuesto por Makinson y Alchourrón (1981), como un modelo posible para explicar características del razonamiento jurídico llevado a cabo por jueces y abogados. En realidad, cuando se procura hacer consistente a un código contradictorio no permanecemos propiamente dicho en el código original, sino que lo reemplazamos por otro, dado que el conjunto que lo constituye tendrá una composición diferente a la del conjunto original. Puede, como se apuntó, que así procedan frecuentemente los juristas prácticos y, quizás, los moralistas casuistas y tiene alto interés teórico explicarlo en un análisis del razonamiento decisorio jurídico, por ejemplo, y de sus formas argumentativas. Queremos mostrar, en cambio, que cabe otra actitud en lo que hace al punto de vista lógico, una actitud lógica distinta, válida para ser aplicada a códigos inconsistentes o contradictorios.

Un código normativo, moral o jurídico, $\mathrm{C}$, puede ser, como advertimos, inconsistente. Dada la interpretación arriba adoptada, estaríamos ante un conjunto de enunciados descriptivos de normas, o, en otra lectura, frente a un metadiscurso normativo, como se suele interpretar al discurso de la metaética 
o de la ciencia dogmática del derecho formada según Kelsen, por Rechtssätze. De quedarnos $\varnothing \div, \mathrm{k}^{\wedge}$ en una lógica clásica, tendríamos las consecuencias contradictorias: $C \mid p$ y $C \mid-p$ donde - es el símbolo de la negación y | el de deducción de la lógica tradicional. Por consiguiente, desde el punto de vista lógico, $C$ sería trivial, puesto que en él es derivable $C \mid q$, donde $q$ es cualquier proposición expresable en el lenguaje $C$ (o, si se quiere, para una interpretación prescriptiva, q sería cualquier norma, en uso prescriptivo, expresable en ese lenguaje).

Empero, no sería posible aplicar la lógica clásica a la sistematización de $C$, sin que tuviéramos que transformarlo cuando $C$ es inconsistente, pero sin trivializarlo. Es cosa clara que, en investigación semejante, suponemos que no se altera $C$ para hacerlo consistente. De hacerse tal cosa, $C$ dejaría de ser el código inicial, como arriba se indicó, puesto que su composición y estructura se verían modificadas o cambiadas. Lo que pretendemos, pese a todo, es mantener la estructura lógica prístina de $C$. Si $C$, por caso fuera una constitución política positiva, deseamos extraer las consecuencias lógicas posibles de $C$, sin convertirla por ello en un texto constitucional consistente, con sus estructuras internas que establecen órdenes jerárquicos, pese a las antinomias debidas a autorreferencias y sin presuponer tampoco que $C$ es trivialy, por ende, que cualquier cosa pueda deducirse de $C$. Por cierto que esto debe lograrse, nos parece, resguardando la índole intuitiva de la noción de consecuencia; o sea: no corresponde desviarse demasiado del concepto tradicional de consecuencia lógica que, justamente ha sido puesto en cuestión en los desarrollos recientes de sistemas expertos jurídicos a partir de lógicas no monotónicas (cf. Atti Firenze 1989, Makinson 1989, Sartor 1991). Para alcanzar ese objetivo, que es una finalidad de la lógica paraclásica, nos atendremos a un concepto de consecuencia deductiva análogo al concepto tradicional, tal como es analizado por Tarski (Tarski 1956).

En este trabajo, además, nos limitaremos exclusivamente al cálculo proposicional, aún cuando el mismo pueda ser extendido fácilmente al cálculo de predicados de primer orden o a lógicas de orden superior. Designaremos con $L$ al proposicional clásico, axiomatizado de alguna de las maneras usuales. $L$ poseerá, por lo tanto, un concepto de deducción representado por el símbolo |.

Representaremos conjuntos de fórmulas con letras griegas mayúsculas, y a las fórmulas, con letras latinas minúsculas. Los conectivos que nos interesan son los siguientes: (implicación material), $\mathrm{v}$ (disyunción), ^ (conjunción), $\mathrm{k}($ negación) y $\varnothing$ (equivalencia material). En la escritura de fórmulas, deducciones, etc., echaremos mano de convenciones obvias que quedarán en claro por el contexto (inclusive en lo que hace al uso de paréntesis).

La introducción luego de ciertos principios básicos de la lógica paraclásica permite verificar de inmediato que puede ser ampliada para obtener lógicas modales, deónticas, temporales, etc. manteniendo todas ellas características 
paraclásicas. Así, pues, la lógica paraclásica abarcaría un gran número de lógicas, similares de cierto modo a las lógicas complementarias de la lógica clásica. Ello interesa sobremanera toda vez que se trate de elaborar una lógica modal apta para reconstruir la noción intuitiva de derivación racional que utilizan los juristas.

\section{La lógica $P$}

Sea $P$ una lógica proposicional cuyos conceptos sintácticos coincidan prácticamente con los del cálculo proposicional clásico arriba descrito. La distinción fundamental entre un cálculo proposicional clásico y $P$ radica en el hecho de que la definición de deducción, o consecuencia deductiva, en $P$ es diferente de la clásica. Se trata de modificar la noción clásica de deducción, corriente en la lógica usual, de manera que obtengamos una nueva noción, | que nos permite operar con sistemas proposicionales contradictorios o inconsistentes, sin riesgo inmediato de trivialización.

En lo que sigue desarrollaremos un estudio sintáctico y semántico de $P$, limitándonos a sus resultados más sencillos, pero fundamentales, sin embargo, para el desarrollo de una lógica deóntica paraclásica.

Definición 1 (consecuencia sintáctica): Sea ) un conjunto de fórmulas, y $P$ una fórmula. Escribiremos: ) | $p$, y diremos que $p$ es una consecuencia estricta o paraclásica de ), si 1) $p 0$ ) ; o, 2) si existe un subconjunto consistente ' , tal que ' $\mathrm{d}$ ), y donde ' $\mid p$ (expresión metalingüística en que el símbolo representa el concepto de consecuencia sintáctica del cálculo proposicional clásico). La noción de consecuencia paraclásica, que podría denominarse «restricta», restringe la noción de consecuencia clásica. En nuestra notación, las nociones de consistencia, inconsistencia, etc., serán las clásicas, mientras no las distingamos con un asterisco (consistencia*, etc.) de las nociones paraclásicas correspondientes.

El conjunto vacío, i , es siempre consistente en la lógica clásica, puesto que de él no podemos derivar, evidentemente contradicción alguna, siendo esa lógica consistente. En una lógica paraclásica, por el otro lado, así como en la mayoría de las lógicas paraconsistentes, el conjunto vacío también se caracteriza porque no cabe derivar de él contradicción alguna. En la lógica paraclásica propuesta modifícase, pues, el concepto de deducción cuyo conjunto de hipótesis es vacío. Las fórmulas válidas en el nuevo cálculo, pues, son las mismas que en el cálculo clásico, pero no son las deducciones válidas. Pretendemos modificar mínimamente el sistema clásico.

Dado, pues, que el conjunto vacío o nulo es consistente, tendremos los siguientes teoremas:

Teorema 1: Si p fuese teorema del cálculo proposicional clásico, entonces $\mid p$. 
Corolario 1: Si p fuese teorema del cálculo proposicional clásico, y ' un conjunto cualquiera de fórmulas, tendríamos que ' $\mid p$.

Un teorema como - " $\div(" \div$ b) es, por lo tanto, fórmula válida en lógica paraclásica. Sin embargo, en ella, de una contradicción, "^_", no cabe deducir cualquier cosa. En lógica paraclásica, de $\{",-"\}$ no cabe inferir cualquier fórmula.

Teorema 2: ) | $p$, siempre que $p 0)$.

Teorema 3: Si D fuera consistente (en el cálculo clásico), tendríamos que ) $\mid p$, si, y sólo si ) | $p$. Por ende, si ' fuera consistente desde el punto de vista de la lógica clásica, sus consecuencias, tanto en lógica clásica, como en lógica paraconsistente, son las mismas.

Estos teoremas establecen las relaciones arriba postuladas entre la lógica clásica y la lógica paraclásica construida.

Teorema 4: ) | $p$ implica ) $C^{\prime} \mid p$, cualquiera sea el conjunto de fórmulas.

Definición 2 (trivial*): ) será trivial* si ) | $q$, para toda fórmula $q$. En caso contrario, ) será no-trivial*.

Teorema 5: Tenemos tanto $\{p,-p\} \mid p$ como $\{p,-p\}$ pero $\{p,-p\}$ es no-trivial*. Esto es, fórmulas $q$ tales que $\{p,-p\} \bigvee q$.

Este teorema, en general, puede ser demostrado así: si admitimos a q como una variable proposicional, tendremos $\mathrm{x}^{*}\{p,-p\} \mid, p$, puesto que $\{\mathrm{p}\}$ es consistente clásicamente y vale que $p \mid p$. Del mismo modo tendremos $\{p,-p\} \mid-p$, dado que también $\{-p\}$ es clásicamente consistente $\mathrm{y}$, por ende, vale que $\mathrm{x}^{*}\{-p\} \mid-p$. En lógica clásica, en cambio, no tenemos ni $\{p\}$ $\mid q$ ni $\{-p\} \mid q$. Por ende $\{p,-p\} \mid$ q. Esta demostración, empero es un tanto genérica, pues vale para toda fórmula del cálculo proposicional.

Cabe demostrarlo, pues, de la siguiente manera: sea " una fórmula cualquiera arbitraria. Si | " (cuando " es una tautología), resulta que existe una \$ no contradictoria tal que " $\$$. Luego, \{", -" $\}$ | \$. Si en cambio, | ", " en cuanto contradictoria lleva a -", y luego entonces $\{",-"\} \mid \$$ (donde $\$$ es interpretada como antes). Si, por fin, siendo " contingente, | -", tendríamos que existe una $\$$ tal que " $\mid$, así como -" | \$. De ahí, siempre y cuando \$ sea variable proposicional que no aparece en ", resultaría $\{",-"\} \mid \$$.

Teorema 6: $\mathrm{Si}\{\mathrm{p}\}|q, \mathrm{y}\{\mathrm{q}\}| r$, entonces $\{\mathrm{p}\} \mid r$. La consecuencia deductiva paraclásica es transitiva.

Definición 3: (operador de consecuencia paraclásica): ) $=\{p:) \mid p\}$. Sin ) es el conjunto de las consecuencias paraclásicas de ), ) cumple en la lógica paraclásica una función semejante al operador de consecuencia de Tarski, $\mathrm{Cn}())$.

Teorema 7: ) d 6. O sea:' d 6 implica que ' d ).

$\mathrm{Si})$ está incluido en el conjunto de sus consecuencias 6 tendremos también que las consecuencias de las consecuencias de ) son consecuencias de ). Como 6 queda definido, a fortiori queda también definido ${ }^{\text {a }}$. $\mathrm{O}$, en el 
simbolismo de Tarski: si se define $\mathrm{Cn}()$ ), para cualquier conjunto ), entonces también queda definido $\mathrm{Cn}(\mathrm{Cn}())$ ). Escribiremos $\mathbf{6} \mathbf{d}=\underline{\mathbf{a}}$ para afirmar que el conjunto de las consecuencias de ) está contenido en el conjunto de las consecuencias de las consecuencias de ) . O sea, según Tarski, $\mathrm{Cn}())$ d $\mathrm{Cn}((\mathrm{A}))$. Sin embargo, en algunos casos, cabe obtener la fórmula más fuerte

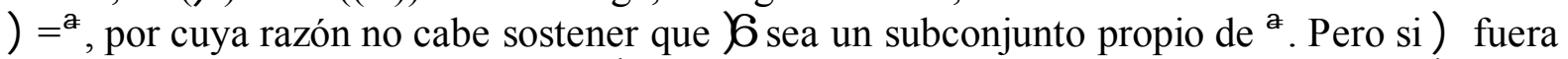
clásicamente consistente, tendríamos $\widehat{W}=\underline{\mathrm{a}}$, como Tarski postula para la lógica clásica. $\mathrm{Si}$ ) fuera inconsistente, ello no resultaría. Puesto que, si $)=\{p,-p\}$; entonces: $p$ v $-q 0), \mathrm{y}-p 0\}$. Por ende, $-q 0$ a y $q 0$ a , aunque resulte que $q 0$ 6. La lógica paraclásica sólo retiene, pues, $\widehat{6} \mathrm{~d}$ ) .

Definición 4: Designaremos T al conjunto de fórmulas de $\mathrm{P}$.

Teorema 8: Si 6 ...T, entonces ) es no-trivial* y viceversa. Vale decir, exiten fórmulas que no son consecuencia paraclásica de ). Esto es: ) es no-trivial*. Este teorema reitera la anterior definición 2.

Teorema 9: Sea ) un conjunto finito de fórmulas; entonces, la relación ) | $p$ es decidible, pues existe un procedimiento mecánico para decidir si $\mathrm{p}$ es o no es consecuencia paraclásica de ). Como es posible obtener los componentes consistentes de ), cabe recurrir a tablas de verdad o procedimientos similares, para decidir la validez de la relación.

Definición 5 (inconsistente*): diremos que ) es inconsistente* si existe una fórmula $p$ tal que ) | $p$ y también ) | $-p$. En caso contrario, diremos que ) es consistente*.

La consecuencia demostrable que nos interesa destacar es:

Teorema 10: Existen conjuntos inconsistentes* que, sin embargo, son no-triviales*.

Definición 6 (componentes de ) ): Sea ) un conjunto de fórmulas. En ) podemos distinguir sus subconjuntos maximales consistentes en ), $\mathrm{si}^{\mathrm{I}}{ }^{\mathrm{C}}$ es consistente y no está contenido propiamente en ningún conjunto consistente $\mathrm{Q}$, tal que $\mathrm{Q} \mathrm{d}$ ) ). Denominaremos a los conjuntos maximales consistentes de ), componentes de ).

Teorema 11: Sea $\mathrm{p}$ una fórmula no contradictoria. Entonces, ) | $p$ si, y solamente si existe un componente ' de $)$, tal que ' $\mid p$.

Teorema 12: $\mathrm{Si}) \mid p$, entonces ) | $p$, pero no necesariamente a la inversa.

Definición 7 (consecuencia semántica paraclásica): Diremos que $p$ es una consecuencia semántica paraclásica de ), y escribiremos ) $\ddot{\mathbb{Q}} p$, si todo modelo clásico de por lo menos un componente de ) es también modelo de $p$, o, si $p$ no tuviera modelo, $p 0$ ) .

Teorema 13: ) | $p$, si, y sólamente si, ) $\ddot{\mathbb{Q}} p$.

Teorema 14: En $P$ tenemos que $6 \stackrel{6}{ } \mathrm{~d}^{\mathrm{a}}$, aunque no siempre se verifique que $6 \mathrm{~d}$ a . Es obvio que $6 \mathrm{~d}$ a . Pero si $)=\{p,-p\}$, donde $p$ es una variable ) será trivial*, como se verifica fácilmente. 
En el cálculo paraclásico tenemos que son teoremas (i.e.: fórmulas válidas) todos los teoremas del cálculo proposicional clásico y solamente ellos. Por consiguiente, la noción de demostración es la misma que la del cálculo clásico. Pero la noción de deducción, en cambio, es distinta. Por ende, tenemos el siguiente.

Teorema 15: No son válidas en lógica paraclásica las siguientes inferencias, (deducciones) clásicas, entre otras:
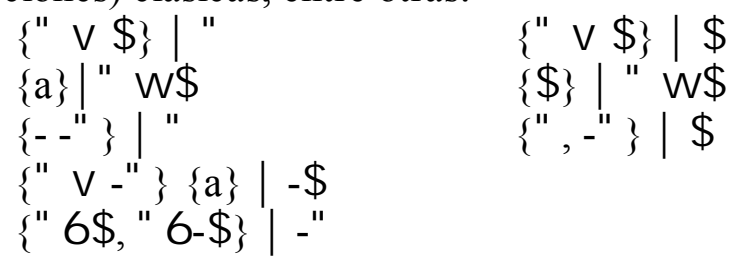

A partir de estos resultados, cabe desarrollar una teoría paraclásica de modelos cuyos detalles, con todo omitiremos aquí.

Es claro que si ) fuera un conjunto de fórmulas, entonces, en mérito a los desarrollos anteriores, podría obtenerse: ) |p,y ) | - $p$, sin que por ello ) quede trivializado por la relación de consecuencia paraclásica. En síntesis, pues, $P$ puede servir como lógica subyacente a sistemas proposicionales inconsistentes y no triviales. En otras palabras, $P$ es una lógica paraconsistente de gran fuerza inferencial. Esquemas inferenciales a que habitualmente recurren los juristas, como la reducción por el absurdo o el argumento e contrario, no quedan excluidos en lógica paraclásica, aunque puedan exhibir formas distintas. Así, si " no es válida clásicamente (i.e., no es una tautología), tendremos que, si ' | "6\$) V ("6-\$) entonces ' | -", una de las formas de argumento por el absurdo válida paraclásicamente.

Es posible, como se indicó, construir una lógica paraclásica de primer orden. Pero el concepto de deducción, en ese caso, no sería efectivo, pues no habría método de decisión para establecer si un conjunto de fórmulas, aún finito, cuenta con un modelo (es consistente). Habrá que examinar este punto en trabajos futuros. Señalaremos que un problema análogo se presenta en relación con la construcción de una lógica paraclásica de orden superior.

[Cabe observar que la definición arriba ofrecida de consecuencia paraclásica depende en rigor de la sintaxis del conjunto. De ahí que, por ejemplo, los conjuntos de fórmulas siguientes son todas clásicamente triviales (contradictorios):

$$
\left\{\begin{aligned}
1_{1} & =\{p, p 6 q, p 6-q\} \\
2_{2} & =\{p \vee(p 6-q), p 6-q\} \\
\}_{3} & =\{p \vee(p 6-q), p 6 q\} \\
{ }_{4} & =\{p \vee(p 6 q) \vee(p 6-q\} \\
{ }_{5} & =\{p,(p 6 q) \vee(p 6-q\}
\end{aligned}\right.
$$

Sin embargo, no sucede lo mismo en la lógica paraclásica. 


\section{Una lógica deóntica paraclásica}

$\mathrm{Si}$ en lugar del cálculo proposicional clásico, partiésemos de un cálculo proposicional deóntico, conforme al método anteriormente expuesto, obtendríamos una lógica deóntica paraclásica. Semejante lógica puede manejar sistemas deónticos inconsistentes, así como códigos normativos en que se presentan dilemas deónticos, sin que por ese motivo se llegue a una trivialización. Por cierto que corresponde atender que se trata de sistemas deónticos descriptivos que exhiben contradicciones, y no de contradicciones entre prescripciones, no definidas en nuestro sistema.

A continuación desarrollaremos una lógica deóntica paraclásica, en elnivel proposicional y mostraremos como la presencia de normas (i.e.: Rechtssätze) contradictorias, o de dilemas deónticos, no hace de su manipulación lógica cosa trivial.

Partiremos de un lenguaje proposicional $L$, como el anteriormente descrito, al que agregaremos el operador deóntico-descriptivo de obligación: $\mathrm{O}$. La noción de fórmula y los demás conceptos de la gramática de $L$ se definen de la manera usual. Por ende, el sistema deóntico paraclásico que llamaremos $T D P$ poseerá los postulados (reglas de inferencia primitivas y esquemas de axiomas) de $T D$ (cuyos axiomas específicos se indican a continuación):

I) $\mathrm{p}$, siempre que $\mathrm{p}$ sea instancia de una tautología (del cálculo proposicional clásico).

II) $\frac{p, p \rightarrow q}{q}$

III) $O(p \rightarrow q) \rightarrow(O p \rightarrow O q)$

IV) $\mathrm{Op} \rightarrow-\mathrm{O}-\mathrm{p}$

V) $\frac{p}{O p}$, cuando $p$ es tesis de $T D$.

En $T D$ se introduce el símbolo de deducción, |, el concepto de teorema etc., de la manera corriente $T D$ es el análogo deóntico del ... modal alético de Feys-von Wright (cf. Hughes \& CressweIl, 1968). Definiremos, entonces, la lótica $T P D$, de manera que TPD esté en relación con $T D$, como $P$ lo está con la lógica proposicional clásica. En este cálculo deóntico $T P D$ tendremos que todo teorema de TD es también teorema de TPD. Lo que se modifica es el concepto de deducción, para poder manejar sistemas inconsistentes sin incurrir en el peligro de trivialización ni en los dilemas deónticos conocidos, así como tampoco en una trivialización deóntica en la cual | $O p$, para cualquier proposición $p$.

En especial, cabe definir sin dificultad la noción de consecuencia sintáctica de TDP, |, cuidando de definir los componentes consistentes de un conjunto de fórmulas, no sólo como consistentes a nivel proposicional, ) $\mathrm{d}^{\prime}$ 
es un componente de ' si 1) D es consistente con respecto a fórmulas proposicionales puras, vale decir, donde no exista un ", fórmula proposicional tal que ) | " y ) | -". Si " no es fórmula proposicional pura, 1) implica 2): ) no implica $\mathrm{O} q$, para cualquier proposición $q$ ).

Se comprueba fácilmente que un código normativo basado en TDP puede ser inconsistente sin ser trivial, y que los dilemas deónticos, como $\mathrm{O} p \vee \mathrm{O}-p$ no hacen trivial a TDP.

Cabe observar también que $P$ se encuentra íntimamente correlacionado con la llamada lógica discursiva de Jaskowski (cf. por ejemplo, da Costa 1975). Interesa también destacar el punto en relación con las llamadas lógicas conversacionales de Joergensen.

\section{Consideraciones generales relacionadas con el derecho, la moral, etc.}

Es frecuente, entre juristas y moralistas, considerar no sólo que el discurso metanormativo de la ciencia jurídica y de la especulación ética está sujeto a pautas lógicas, sino que, a nivel prescriptivo, las decisiones morales y jurídicas pueden ser racionales en la medida en que están sujetas a pautas lógicas. Juristas y moralistas hablan corrientemente de contradicciones normativas, cuando encuentran reglas prescriptivas prima facie incompatibles, así como inferencias normativas, cuando a partir de normas generales se deducen normas específicas. La teoría jurídica, en especial, se ha preocupado por la reconstrucción racional del razonamiento práctico mediante el cual los órganos decisorios llegan a conclusiones prescriptivas que consideran consecuencia lógica de premisas declarativas y normativas. Cual sea, sin embargo, la lógica o lógicas que legitimen la validez de tales razonamientos en contextos normativos o decisorios no es asunto pacífico. Por el contrario, es frecuente entre teóricos del derecho, como H. Kelsen, y filósofos y lógicos, como G. H. von Wright, rechazar la posibilidad de que, en esos contextos normativos y decisorios, quepan razonamientos deductivos en sentido lógico tradicional, toda vez que las normas en función prescriptiva no admiten un cálculo veritativo-funcional como el de la lógica clásica. Frente a este irracionalismo normativo, como se lo ha denominado, los juristas y los moralistas, desde siempre, consideran que es posible obtener derivaciones deductivas en un discurso normativo, ateniéndose a pautas lógicas.

La lógica clásica proposicional, como es sabido, y las lógicas modales deónticas elaboradas a partir de dicho cálculo, exhiben teoremas paradójicos, por lo menos, en el sentido de que no se ajustan a las interpretaciones corrientes de los lenguajes naturales. Así, en terreno de una lógica normativa, la paradoja de Ross deriva del banal teorema que permite inferir una disyunción a partir de una proposición cualquiera. El principio ex falso aliquid sequitur que vale como teorema clásico, tendría su equivalente en los sistemas deónticos standard, interpretándoselo en el sentido de que cabe cualquier 
conclusión normativa a partir de premisas deónticas contradictorias. En contextos decisorios, la validez ilimitada de este último teorema tendría consecuencias graves. Las sentencias judiciales, en los estados de derecho, pretenden ser consecuencia razonada, entre otras cosas, de las normas jurídicas reconocidas como válidas. Si un orden jurídico, como sucede en los derechos positivos históricos, contuviera normas contradictorias, cualquier decisión judicial, sea cual fuere su contenido, aparecería como una derivación deductiva correcta del derecho vigente. Tal consecuencia es rechazada por los juristas tradicionales y también por las ideologías políticas que respaldan el llamado estado de derecho. Aceptar esa consecuencia parece incurrir en una forma de irracionalismo decisorio.

Claro que para las tendencias irracionalistas mencionadas, para las cuales es imposible definir estrictamente una contradicción normativa, esa aparente paradoja del principio de Duns Scotus (también atribuido al Pseudus Scotus) se desvanece. Pero el precio pagado -el discurso prescriptivo del derecho y de la moral sería alógico y, por ende, irracional-, parece demasiado alto. Es corriente que los juristas tengan que hacerse cargo de códigos normativos en que, prima facie, las contradicciones deónticas abundan: basta recorrer el articulado de cualquier ley compleja o intentar hacer explícitas las reglas morales vigentes en una comunidad. Más aún: los juristas profesionales saben que en toda situación conflictiva, como un pleito o litigio, en que debe tomarse una decisión, frecuentemente enfrentarán no sólo premisas fácticas contradictorias (como las versiones contrarias de algunos hechos que darán en un conflicto judicial las partes litigantes), sino normas inconsistentes que el derecho positivo contiene como resultado no sólo de incoherencias legislativas, sino de la acumulación histórica caótica de leyes y normas. Los juristas han desarrollado estrategias para solucionar estos problemas, como la regla de la lex posterior y otras. Recientemente se ha planteado la cuestión de si la aparición de paradojas lógicas en el razonamiento jurídico no fuera el resultado de la aplicación de una lógica, como la clásica, inadecuada para el control racional del proceso decisorio judicial o político. Por ende, se han propuesto lógicas normativas divergentes destinadas a evitar tales paradojas y, en especial, las consecuencias teóricas catastróficas del teorema de Duns.

El pensamiento especulativo moral, en cambio, no ha desarrollado técnicas semejantes, aunque ha reconocido desde siempre la existencia de paradojas en el discurso normativo ético, sobre todo bajo la forma de dilemas morales. Carece de sentido, por cierto, establecer una regla moral semejante a la regla jurídica de la lex posterior, toda vez que se suele pensar a las normas o principios morales como atemporales. Otras propuestas destinadas a disolver las inconsistencias presentes en códigos morales son, en todo caso, no sólo cuestionables desde un punto de vista lógico, sino que carecen propiamente de fundamento ético que las convalide (cf. R. Routley \& 
V. Plumwood, 1984). En efecto, una regla de preferencia en el plano metaético no puede ser considerada un precepto moral en sentido corriente.

Las lógicas sugeridas para evitar las consecuencias contraintuitivas de la lógica clásica en el terreno normativo, han sido de distinto tipo. Algunas intentan una reconstrucción de las modalidades efectivas con que los juristas razonarían al adoptar decisiones normativas. Tendríamos algo así como una lógica jurídica ad hoc, como la subyacente a ciertos argumentos jurídicos (a simile, e contrario, a fortiori) que no son, en principio, leyes lógicas válidas. (cf. Perelman 1976, Miró Quesada, 1988). Otros han sugerido lógicas más dúctiles o blandas, que admiten más valores designados que la verdad y falsedad clásicas (cf. Weinberger 1987); se trata, por caso, de lógicas plurivalentes o lógicas que eliminan algunas de las restricciones que imponen el tercero excluido o las tautologías correspondientes al condicional material. Tenemos, así, por ejemplo, lógicas naturales deónticas, con formas de deducción natural o por secuencias, o lógicas relevantes normativas (cf. Alchourrón \& Martino 1989). O bien se proponen recurrir a una lógica deóntica fuzzy para hacerse cargo de conceptos jurídicos y razonamientos por analogía (Reisinger 1979, Mazzarese 1992). Una versión más radical, que pone en cuestión la noción misma de racionalidad, es la lógica deóntica paraconsistente no alética diseñada por $\mathrm{N}$. Grana (Grana 1990a y 1990b).

También se ha planteado el interrogante de si los razonamientos jurídicos decisorios, en contextos normativos, considerados como deducciones, responden a las condiciones clásicas de la noción de consecuencia. Esta última responde al requisito de monotonía, como lo expresa típicamente la regla inferencial que permite agregar premisas al antecedente de todo condicional material. Dicha característica, propia de la noción de consecuencia de la lógica clásica, ha sido puesta en cuestión por lógicos interesados en reconstruir el razonamiento jurídico. Se han propuesto así lógicas no monotónicas, donde la introducción de nueva información o premisas en los antecedentes de una conclusión deductiva quitan validez al razonamiento. Tal cosa ha sido propuesta, muy especialmente, por programadores interesados en la construcción de sistemas expertos en derecho (cf. Smith, 1989), así como su necesidad ha sido cuestionada (cf. Makinson, 1989). Se trata, pues, de un problema aún pendiente (cf. Alchourrón, 1991).

Otra alternativa teórica interesante, empero, se encuentra en recurrir, ya no en lógicas divergentes de tipo natural o relevante, sino paraconsistentes, lógicas en las cuales la inconsistencia (presencia de contradicciones, definidas de cierta manera), no implica la trivialización que el principio ex falso introduce en la lógica clásica. En ésta, inconsistencia y trivialización se complican. ¿Es posible, en cambio, construir sistemas lógicos inconsistentes, pero no triviales? Ello ha sido logrado, en extensa medida, por los sistemas lógicos paraconsistentes que desarrollarán, inicialmente, Jaskowski y, más recientemente, N. da Costa y sus discípulos (cf. N. Rescher \& Brandom, 1979 y Arruda, cit. supra, 1980). Tales lógicas paraconsistentes 
no sólo tienen importancia para el desarrollo de la noción filosófica de verdad (cf. von Wright, 1990), un concepto central en el pensamiento filosófico tradicional y para la lógica clásica, sino que han sido extendidas a lógicas modales, como las deónticas que procuran establecer sistemas deductivos para lenguajes prescriptivos. Las que han sido desarrolladas permiten interesantes extensiones a tradicionales problemas del derecho y la moral, como es el caso de los dilemas morales, los sistemas mixtos jurídico-morales y los conflictos normativos (cf. inter alia, Puga \& da Costa 198., Puga \& da Costa \& Vernengo 1990, 1991 y Grana 1991). Se ha recurrido a las mismas para afinar el análisis de ciertas nociones jurídicas no suficientemente claras en el marco del pensamiento lógico tradicional, clásico y deóntico standard. Otra cosa, cabe advertir, es la reconstrucción formal de las formas habituales de argumentar que los juristas emplean. Por de pronto, en esas líneas de pensamiento el status teórico de las reglas de argumentación propuestas distan de ser claras o, en todo caso, difieren grandemente de los procedimientos inferenciales de las lógicas. Se trata, por lo común, de argumentos persuasorios destinados a justificar decisiones, cuya lógica implícita es nuevamente la clásica (Alexy 1978/83, Atienza 1991).

En rigor, este vasto abanico de teorías construidas para explicar (en sentido carnapiano) las formas del razonamiento decisorio, sobre todo en terreno jurídico, intentan salvaguardar por lo menos alguna concepción de racionalidad que permita entender actos sociales de gran importancia, como es el funcionamiento de un sistema institucional judicial destinado a solucionar ciertos conflictos, como acciones previsibles, i.e.: racionales en sentido weberiano. Ello, pareciera, tiene algún interés analítico. Las lógicas arriba mencionadas no cumplen primariamente tales funciones, siendo construcciones conceptuales puramente teóricas. Sin embargo, pueden adquirir una relevancia filosófica enorme. No sólo permiten análisis mucho más finos de ciertos problemas que desde siempre han intrigado a la filosofía, si no que ponen en cuestión temas básicos de la investigación filosófica en el terreno ético y jurídico: la noción de norma, de consistencia decisoria, de voluntad racional, de un legislador racional, de racionalidad práctica, entre otras. No cabe aquí entrar en estos temas perdurables y reiterados.

\section{La lógica deóntica paraclásica y el razonamiento jurídico}

Los sistemas sugeridos en la presente nota, una lógica paraclásica proposicional y una lógica deóntica paraclásica, parten de una nueva propuesta de definición de la noción de consecuencia deductiva. La misma no difiere del concepto clásico de consecuencia, en cuanto continúa siendo reflexiva, transitiva y monotónica (cf. supra teoremas 4 y 6). Empero, no permite la equiparación de la inconsistencia de un sistema con su trivialidad deductiva (teorema 10). Esto es, el sistema no permite derivar paraclásicamente 
tanto una norma $p$ como su negación - $p$. La inconsistencia estricta de un sistema normativo se da cuando tanto una norma, como su negación, son consecuencia estricta (paraclásica) de un conjunto de enunciados. Este tipo de inconsistencia, por la definición misma de consecuencia estricta, no permite el análogo de la deducción ex falso. En efecto, el conjunto de enunciados que son premisas de la consecuencia estricta son un subconjunto clásicamente consistente del universo de discurso ). Cabe, pues, considerar que nuestra definición de consecuencia lógica difiere de la tarskiana, en el sentido de que la restringe. Por ejemplo, de acuerdo con Tarski, tendríamos $\{",-"\} \mid$ \$, para cualquier enunciado \$. Formalmente, por el otro lado, conforme con Tarski, tenemos: $\mathrm{Cn}(\mathrm{Cn}()))=\mathrm{Cn}(())$. En la lógica paraclásica sólo mantiene, como se indicó, $6 \mathrm{~d}$ ) .

Ahora bien, conviene establecer con alguna precisión cuáles son los contextos en que la derivación ex falso preocupa a los juristas. Estos rara vez tienen que tratar con normas llamadas categóricas, del tipo en que se suelen enunciar principios morales (p. ej.: «prohibido matar», «obligatorio cumplir las promesas», etc.) El modelo canónico de una norma jurídica es, más bien, un enunciado condicional en el cual a ciertos antecedentes fácticos se le atribuye o imputa (recurriendo al término kelseniano de Zurechnung) dice (en su versión descriptiva), o dispone (en su versión prescriptiva), que dados ciertos hechos alguna conducta es obligatoria (tomando al functor o como modalidad deóntica primitiva). Dos normas categóricas, como $\mathrm{O} p$ y -O $p$ (o las normas contrarias $\mathrm{O} p$ y $-\mathrm{O} p$ ) parecen clásicamente inconsistentes. Pero la inconsistencia de normas condicionales reviste otras formas. Los juristas pensarían que son inconsistentes normas que para los mismos hechos antecedentes tienen consecuencias deónticamente contradictorias. Pero no es éste el caso típico en que el jurista o el moralista tienen que enfrentar datos inconsistentes.

Para el caso mencionado -hechos antecedentes idénticos y consecuencias normativas contradictorias-, los juristas cuentan con recursos reglados (esto es: que el propio sistema contiene como solución prescriptiva preferida) como la regla lex posterior. La situación característica en que el jurista enfrenta datos inconsistentes se da, realmente para los órganos decisorios, e idealmente para el jurista teórico, en un conflicto social con varios intervinientes, en que se esgrimen hechos antecedentes incompatibles como condicionantes de cierta solución normativa, $\mathrm{o}$ hechos antecedentes condicionantes de soluciones normativas pretendidas incompatibles. En todo litigio alguna parte afirma el hecho A y la parte contraria lo niega, o una pretende la aplicación de la norma $\mathrm{N}$, mientras la otra aspira a que se aplique una norma incompatible con N. El juez, pues, tiene que decidir ante una situación en que se postula a A y -A como antecedentes de alguna consecuencia normativa única, o una situación en que frente a un conjunto de hechos aceptados se pretende, por una y otra parte, la aplicación de la norma $\mathrm{N}$ y de la norma -N. 
Pareciera que el juez se encontrara ante un dilema insoluble. La técnica decisoria judicial consiste justamente en disolver, como se suele decir, la contradicción que las partes mantienen, por caso, con respecto de los hechos que condicionan una consecuencia normativa. El juez, pues, rechazará la verdad invocada, y no la imposible verdad empírica, de hechos como A y -A, para poder justificar racionalmente su decisión. Pero, antes de llegar a esa decisión final, el juez tendrá que hacer frente a un contexto en que la decisión sobre la verdad de A y -A tiene que ser adoptada. El juez, en esa etapa previa, razonaría (podríamos reconstruir formalmente su razonamiento) de manera tal que la inconsistencia de las premisas no lo lleve de antemano, ex falso, a una consecuencia cualquiera. Seguramente no es tal la actitud psicológica del juez, quien seguramente supone que ciertos hechos son verdaderos y otros falsos. Pero cabe pensar que la inconsistencia de las premisas sobre los hechos no constituye de por sí un impedimento para llegar a conclusiones racionales, sea que las contradicciones son puestas de lado o desdeñadas, sea que el juez no considera que las alegaciones incompatibles de las partes lo lleve necesariamente a consecuencias arbitrarias, como en el caso de las inferencias ex falso. Esa situación puede ser analizada, creemos, recurriendo a un cálculo paraconsistente.

Conviene advertir, con todo, cuáles son los costos y beneficios de semejante estrategia analítica. El riesgo de trivialización que una lógica clásica veritativo-funcional y una lógica deóntica standard suscitan proviene, claro está, de las interpretaciones que la implicación material ocasiona como forma lógica impuesta a la reconstrucción de hipótesis normativas, como son las obligaciones condicionales, forma que la teoría general del derecho atribuye a las normas jurídicas. Parece claro que la implicación material no satisface los requisitos intuitivos del pensamiento normativo ejercitado por juristas y moralistas. De ahí que, desde temprano que propusieran otras interpretaciones, desde la oscura idea kelseniana de un condicional imputativo (Zurechnung), a condicionales modales o contrafácticos (McCarty 1987, Alchourrón, 1986). Tales propuestas, sin embargo, no han resultado satisfactorias en cuanto no excluyen enteramente consecuencias paradójicas, por lo menos para las formas de pensar corrientes entre los juristas, al punto de plantearse dudas sobre la validez misma del modus ponens en contexto normativos, una regla definitoria casi de la noción misma de inferencia lógica.

Puede, en ese respecto, considerarse una ganancia que, recurriendo a esas estrategias formales, se eviten paradojas derivadas de la implicación material, entre ellas, las derivadas de la consecuencia ex falso. Algunas de las lógicas deónticas paraconsistentes construidas a ese efecto producen ciertamente ese beneficio teórico. Pero, para todas ellas, el costo reside en la eliminación, como formas válidas de inferencia, de ciertas derivaciones tradicionales. Así por ejemplo, en el sistema $B^{D}$ (Grana 1990), tenemos que los análogos deónticos del principio de contradicción, del argumento por el 
absurdo, del tercero excluido, de la doble negación interna, así como el principio de Duns, están excluidos como tesis inválidas en el sistema. Ahora bien, cabe preguntarse si esta forma heroica de evitar el riesgo de trivialización normativa no empobrece excesivamente el instrumental deductivo a que el jurista podría recurrir y que no refleja suficientemente las formas inferenciales que utiliza inocentemente. Y, filosóficamente, cabe preguntar si la novedosa noción de racionalidad que de esa suerte se introduce, aunque permita encarar derivaciones lógicas en códigos normativos inconsistentes e incompletos, no lleva a preferir un sistema lógico que sólo sea capaz de reconstruir un segmento menor del discurso racional latu sensu de los juristas. No se advierte, en cambio, cómo un sistema lógico semejante sea preferible para reconstruir nociones puramente teóricas, como la de la Grundnorm kelseniana (según sugiere Grana), un concepto que requiere ser manejado en el metalenguaje de la teoría jurídica con una lógica más fuerte que la lógica paraconsistente y paracompleta a que se recurriría en el lenguaje normativo objeto. Von Wright, en ese respecto, observaba que, en general, la metateoría de teorías inconsistentes o paracompletas se elabora con lógicas clásicas típicas (von Wright 1989).

Es a la luz de estas consideraciones que conviene juzgar las ventajas y desventajas de una lógica deóntica paraclásica, como la propuesta arriba en la sección 3. La misma, permite especialmente operar con conjuntos normativos inconsistentes, pero no triviales, conjuntos que muchas veces pueden servir como modelos aptos para reconstruir el orden normativo que un jurista tiene presente al tomar decisiones. Para cumplir esa función, define (definición 6) componentes, esto es, subconjuntos maximales consistentes cuyos elementos son, en el cálculo paraclásico deóntico, enunciados o fórmulas normativas descriptivas. Estos componentes -un subconjunto maximal consistente incluido en el universo del discurso-, permiten definir una relación paraclásica o estricta de consecuencia (teorema 11) y, luego, una noción de consecuencia semántica (definición 7). En el cálculo paraclásico deóntico se postulan, como esquemas de axiomas y reglas de inferencia, los principios de distribución del operador deóntico O y de subalternación del operador de permisión débil, así como la regla del modus ponens y el principio de extensionalidad que extiende las leyes tautológicas del cálculo clásico al dominio normativo. Se trata, pues, de una lógica que, frente a otras propuestas, cuenta con gran poder o fuerza deductiva. Baste advertir que, en virtud del teorema 1, las tautologías clásicas valen como consecuencias paraclásicas, mientras que, a la inversa, sólo cabe derivar consecuencias consistentes no paraclásicas de consecuencias paraclásicas válidas (teorema 3). Ello, en cuanto el análogo de la derivación ex falso, permite derivar, por cierto, los elementos componentes de la contradicción, pero no cualquier enunciado, lo que haría trivial al sistema (teorema 5).

En suma: las derivaciones clásicas son, en principio, válidas también para el cálculo paraclásico, excluyendo la posibilidad de trivialización. Como 
lógica implícita para la reconstrucción del razonamiento jurídico, el sistema $T D P$, parece, pues, más potente que algunas de las lógicas ad hoc o paraconsistentes y paracompletas propuestas. En TDP, cabe advertir, el tercero excluido deóntico, $\mathrm{O} p \mathrm{v}-\mathrm{O}-p$, equivalente a la ley de subalternidad deóntica: $\mathrm{P} p$ v - $\mathrm{P}-p$, vale, aunque no su forma fuerte paraclásica para representar la modalidad libre o facultativa: $\mathrm{P} p, \mathrm{P}-p$. En ese respecto, nos acercamos quizás a una acertada reconstrucción formal del razonamiento puramente intuitivo del jurista, estableciendo mediante su componente maximal consistente algo parecido al subconjunto minimal, privilegiado o crítico, que también fuera sugerido para esa reconstrución (Alchourrón 1986), sin requerir entrar en la consideración de los órdenes jerárquicos con que los juristas suelen pensar el material normativo promulgado.

En especial, los componentes consistentes del sistema TDP permiten establecer que aquellos casos en que aparecen obligaciones bajo condiciones contradictorias, la solución puede ser derivada como consecuencia del subconjunto formado por las condiciones consistentes, evitándose la trivialización resultante de admitir que todo es obligatorio bajo una condición imposible, como serían condiciones en contradicción clásica. Ello refleja adecuadamente la estrategia corriente de los jueces, quienes frente a datos fácticos y normativos contradictorios, elaboran un subsistema consistente, dentro del conjunto seguramente caótico de hechos y normas invocadas, subconjunto ad hoc esencial que les permite alcanzar una derivación racional de la solución que, en derecho estricto, finalmente adopten.

Los autores dedican este trabajo a la memoria de Carlos E. Alchourrón, de quien recibieron observaciones importantes para el desarrollo de la investigación. Desean también dejar constancia de su agradecimiento a M. Coniglio (Buenos Aires) y O. Bueno (Sa\#Paulo) por sus comentarios y críticas a una primera versión de este ensayo.

\section{BIBLIOGRAFÍA}

Alchourrón, C. E., Logic of Norms and Nogic of Normative Propositions, Logique et Analyse 12, Bruxelles, 1969.

-Conditionality and the representation of Legal Norms, in Automated Analysis of Legal Texts: Logic, Information, Law, Elsevier Science Pu., North-Holland, 1986.

-Philosophical Foundations of Deontic Logic and the Logic of Conditionals, Ms., Amsterdam 1991.

-Defeasible, Strict and Sounterfactual Conditionals - Nonmonotonic Inference Relations, en G. Crocco, L. Fariñas del Cerro y A. Heriz (de.), «Conditionals and Artificial Intelligence», Oxford U. Press, 1994. 
-Concepciones de la lógica, en «Lógica», Enciclopedia Iberoamericana de Filosofía, Consejo de Investigaciones Científicas, Barcelona, 1993.

-On Law and Logic, Actas del $17^{\circ}$ Congreso de la I.V.R., Bologna, 1995.

Alchourrón, C. E. \& Makinson, D., Hierarchies of Regulations and their Logic, en Hilpinen, 1981.

Alchourrón, C. E. \& Martino, A., Lógica sin verdad, an Theoria, 7/9, San Sebastián, 1988.

Arruda, A. I., A Survey of Paraconsistent Logic, en Mathematical Logic in Latin America, ed. A.I. Arruda, R. Chuaqui, N.C.A. da Costa, ed., North-Holland Publishing Co., 1980.

C.N.R., Atti (pre-proceedings) del III Convengno internazionale de logica, informatica e diritto, Florencia, 1989.

da Costa, N.C.A., Remarks on Jaskowski's Discursive Logic, Reports on Mathematical Logic 4, 1975.

-The Philosophical Import of Paraconsistent Logic, en Journal of non-classical logic, 1982.

-A New Approach to Deontic Logic, ms., 1989.

da Costa, N.C.A. \& Carnielli, W., On Paraconsistent Deontic Logic, en Philosophia 16, 1986.

da Costa, N.C.A. \& Subrahmanian, YS., Paraconsistent Logic as a Formalism for Reasoning about Inconsistent Knowledge Bases, Instituto de Estudos Avançados, Universidade de S\# Paulo, 1989.

Grana, N., Lógica deóntica paraconsistente, Liguori Editore, Napoli, 1990.

-Contraddizione e incompletezza, Liguori Editore, Napoli, $1990 \mathrm{~b}$.

Hilpinen, R. (ed.), New Studies in Deontic Logic, D. Reidel Pub. Co., Dordrecht, 1981.

Hughes, G. H. \& Creswell, M.J., An Introduction to Modal Logic, Methuen, 1968.

Kelsen, H., Recht un Logik, Wien, 1965.

-Allgemeine Theorie der Normen, Wein, 1979.

Loparic, A. \& Puga, L.Z., Two Systems of Deontic Logic, ms.

Makinson, D., General Pattems in Nonmonotonic Reasoning, en D. Lehmann (ed.) «Nonmonotonic and Uncertain Reasoning», 1989.

Mazzaresse, T., Fuzzy Logic and Judicial Decision-Making, en Actas Simposio sobre derecho, moral y lógica, Córdoba, Argentina, 1992.

McCarty, L. T., Permissions and Obligations, en Atti II Convegno internazionale su logica, informatica e diritto, Florencia, 1985, t. II.

Miró Quesada, F, Ensayos de filosofía del derecho, Universidad de Lima, 1986.

-Lógica jurídica idiomática, en Conferencias III Congresso Brasileiro de Filosofia do Direito, Jo\# Pessoa, 1988.

Opalek, K., Theorie der Direktiven und der Normen, Springer, New York-Wien, 1986.

Perelman, Ch., Logique juridique, París, 1976.

Puga, L.Z. \& da Costa, N.C.A., Logic with Deontic and Legal Modalities: Preliminary account, Bulletin Section Logic, Polisch Academy of Sciences, 2, 1986.

-Lógica deôntica e direito, Boletim da Sociedade Paranaense de Matemática, 2, 1987.

-Sobre a lógica deôntica natássica, en Crítica XIX, 55, UNAM, México, 1987. 
Puga, L. Z., da Costa, N.C.A. \& Vernengo, R.J., Derecho, moral y preferencias valorativas, en Theoria, V, 12/13, 1990.

-Lógicas normativas, moral y derecho, en Crítica, XXIII, 60, México, 1991.

Reisinger, L., Zur Struktur der Analogie im Rechtsdenken, en «Argumentation und Hermeneutik in der Jurisprudenz», Rechtstheorie, Beiheft I, Berlin, 1979.

Rescher, N. \& Brandom, R., The logic of Inconsistency, Rowman \& Littiefield, New Jersey, 1979.

Routley, R. \& Plumwood V., Moral Dilemmas and the Logic of Deontic Notions, Discussion Papers on Environmental Philosophy 6, Departments of Philosophy, Australian National University, 1984.

Sartor, G., Le applicazioni giuridiche dell'intelligenza artificiale: la representazione della conoscenza, Milano, 1990.

Smith, M., The Formalization of Legal Reasoning, en Proceedings of the III International Conference on Logica, Informatica e Diritto, Florencia, 1989.

Tarski, A., Logic, Semantics, Metamathematics, Oxford, 1956.

Vernengo, R.J., Sobre algunas relaç lógicas entre sistemas normativos jurídicos e morais, en Revista Brasileira de Filosofía, XXVIII, 155, S\# Paulo, 1989.

-Racionalidad jurídica y lógicas normativas, en Análisis Filosófico, X, 2, Buenos Aires, 1990.

-Legal Rationality and Divergent Normative Logics, Festschrift f. K. Opalek, Duncker \& Humblot, Berlin, en prensa.

Weinberger, O., Ex falso quodlibet in der deskriptiven und in der präskriptiven Sprache, en Rechtstheorie 6, 1975.

-Kelsens These von der Unadwendbarkeit logischer Regeln auf Normen, en Die Reine Rechtslehre in wissenschaflicher Diskussion, H. Kelsen Institu, Wien, 1982.

-Der normenlogische Skeptizismus, en Rechttheorie 17, 1986.

von Wright, G.H., Norms, Truth and Logic, en Practical reason, Oxford 1983.

-Logiche della verità, en Informatica e diritto XV, Milano, 1989. 